\title{
A simple, self-referenced, intensity-based optical fibre sensor for temperature measurements
}

\author{
Ricardo M. Silva $^{\text {a }}$, J.M. Baptista ${ }^{\text {a,b }}$, J.L. Santos ${ }^{\mathrm{a}, \mathrm{c}}$, A.B. Lobo Ribeiro ${ }^{\text {d,* }}$, F.M. Araújo ${ }^{\text {a }}$, \\ L.A. Ferreira ${ }^{\mathrm{a}}$, O. Frazão ${ }^{\mathrm{a}}$ \\ ${ }^{a}$ INESC-Porto, Rua do Campo Alegre 687, 4169-007 Porto, Portugal \\ ${ }^{\mathrm{b}}$ Exact Sciences and Engineering Competence Centre, University of Madeira, 9300-390 Funchal, Portugal \\ c Faculty of Sciences, University of Porto, Rua do Campo Alegre, 687, 4169-007 Porto, Portugal \\ d Faculty of Health Sciences, University Fernando Pessoa, Rua Carlos da Maia, 296, 4200-150 Porto, Portugal
}

\section{A R T I C L E I N F O}

\section{Article history:}

Received 17 February 2012

Received in revised form

30 October 2012

Accepted 1 November 2012

Available online 21 November 2012

Keywords:

Intensity optical fibre sensor

Fibre Bragg grating

Temperature

\begin{abstract}
A B S T R A C T
A sensing configuration based on an intensity optical fibre sensor for temperature measurement is reported. Two sensing heads, with identical geometrical configuration, connected in series are implemented. Each sensing head is placed between two fibre Bragg gratings (FBGs), being able to provide a self-referenced measurement, and thus eliminate errors that may arise from undesired intensity fluctuations in the configuration. The first FBG, placed before the aluminium tube, acts as the reference FBG, while the second FBG, placed after the aluminium tube, acts as the signal FBG. It is observed that the amplitude of the signal FBG decays when temperature increases, due to the increase of the ferrules' gap and as result of the material thermal expansion. The temperature response has a behaviour that corresponds to a polynomial fit of third order. The crosstalk between the two sensing heads in series is analysed. The temperature sensitivities obtained in the intervals regions of [36, 48.5] ${ }^{\circ} \mathrm{C}$ and $[64,85]{ }^{\circ} \mathrm{C}$ are $2.67 \times 10^{-3}{ }^{\circ} \mathrm{C}^{-1}$ and $1.74 \times 10^{-4}{ }^{\circ} \mathrm{C}^{-1}$, respectively. Ten sensing heads with this configuration can be multiplexed in series network topology.
\end{abstract}

(c) 2012 Elsevier B.V. All rights reserved.

\section{Introduction}

Optical sensors can offer nondestructive and noncontact measurements in several applications such as military, industrial, automation and biomedical. In this field, optical fibre sensors have an important role as sensing elements. The main advantages of using optical fibres are their small size, lightweight, ruggedness, light guiding capacity, flexibility, immunity to electromagnetic field, environmental robustness, low transmission loss and large optical bandwidth [1]. Optical fibre sensors are able to measure with great precision a wide variety of physical parameters such as strain, displacement, pressure [2], temperature [3], humidity [4], electric current [5] and flow [6]. Intensity based optical fibre sensors can offer low cost and simple technologic solutions, ensuring a large range of applications. The main drawback of this kind of sensor is the occurrence of intensity fluctuations from the optical source, and/or the fibre components (such as, fibre couplers, etc.) along the optical system. To ensure accurate measurements, the implementation of a reference mechanism is essential. This reference mechanism provides a

\footnotetext{
* Corresponding author. Tel.: + 351225074630 .

E-mail address: ablobo@gmail.com (A.B. Lobo Ribeiro).
}

degree of insensitivity to source intensity fluctuations and to variable transmission losses in the configuration [6].

Fibre Bragg gratings are generally interrogated by the wavelength shift, but they can also be used as intensity devices [7]. To provide self-referenced measurements, Melle et al. [8], described a passive measurement technique for determination of the narrow-band of guided-wave fibre Bragg grating (FBG). The setup consisted in splitting the back-reflected light from the FBG into two beams. One is filtered in proportion to its wavelength, while the other beam was used as reference to compensate any intensity fluctuations. This detection system was used to measure absolute strain. Later on, in 1993, the same group reported a fibreoptic strain gauge system for use in structural monitoring and smart-structure applications [9]. They used a FBG to measure strain, altogether with a passive wavelength demodulation system to determine the narrow-band, back-reflected spectrum from the grating. This fibre-optic gauge system allowed the measurement of static and dynamic strains with noise-limited resolution. In 1994, Davis et al. [10], demonstrated the use of a simple all-fibre implementation of the wavelength discrimination principle using a wavelength dependent $2 \times 2$ fibre optic coupler. In this system an FBG was used as strain sensor. In 1995, Cavaleiro et al. [11] demonstrated a simple referencing scheme that uses two identical FBGs (one in the sensor head and the other in the signal processing 
region) wavelength-tuned to each other by an electronic servo loop. This innovative referencing scheme works with all types of reflective intensity based fibre optic sensors.

Recently, Wilson et al. [12], proposed a intensity-based technique for interrogation of FBGs sensors. The configuration used two longitudinal modes of a laser diode as a dual-wavelength source to illuminate the FBGs sensors. The main advantage of using such demodulation technique was the reduced complexity and the overall cost, associated with fabrication of an array of identical FBGs.

In the work presented here, the sensing configuration is simple and consists of using an intensity-based temperature sensing head between two fibre Bragg gratings, which do not need to be identical in their central wavelength. One FBG is used as reference signal and is physically located before the sensing head, while the other FBG is spliced after the sensing head and acts as the sensing signal. When the intensity sensing head is subjected to the external physical parameter, the reflected intensity of the second FBG varies accordingly. The intensity-based temperature sensing head is based on a metallic tube involving two geometrically aligned, angled physical contact (APC) ferrules. To interrogate the intensity sensor, the reflected signals from the two FBGs are separated by WDM fibre couplers and detected by PIN photodetectors. The sensing system under study comprehends two selfreferenced intensity-based temperature sensing heads, addressed independently by wavelength separation.

\section{Experimental setup}

Fig. 1 shows the experimental setup, which consisted in a broadband optical fibre source (an Amplified Spontaneous Emission fibre source) with a central wavelength emission at $1540 \mathrm{~nm}$, optical bandwidth of $40 \mathrm{~nm}$ (FWHM) and output power of $\sim 100 \mathrm{~mW}$, an optical fibre circulator (OC), four FBGs (whose reflected power is designated by $P_{1}, P_{2}, P_{3}$ and $P_{4}$, respectively), four WDM add-drops, four identical PIN photodetectors (EXFO IQ 203 ) and two intensity-based sensing heads $\left(S_{1}\right.$ and $\left.S_{2}\right)$. The FBGs had a central wavelength of $1542 \mathrm{~nm}, 1548 \mathrm{~nm}, 1555 \mathrm{~nm}$ and $1561 \mathrm{~nm}$, respectively. The FBGs were written in a standard single mode fibre (SMF-28) by the phase mask technique with an ultraviolet (UV) excimer laser emitting at $248 \mathrm{~nm}$. The optical fibre transfers the reflected signals to the four detectors to enable analysis of the signals from sensors, $S_{1}$ and $S_{2}$. Each WDM adddrop permitted the wavelength isolation of each FBG according to the telecommunications spectral bands $C(1541 \pm 1.6 \mathrm{~nm}), D$ $(1547 \pm 1.6 \mathrm{~nm}), E(1554 \pm 1.6 \mathrm{~nm})$ and $F(1560 \pm 1.6 \mathrm{~nm})$. The isolation losses were $35 \mathrm{~dB}$ and the spectral bandwidth was $5 \mathrm{~nm}$ for all WDM add-drop filters. Each sensing head was composed by two optical fibre APC ferrules, one ceramic sleeve to align the two ferrules, and an aluminium tube holding the complete set, as

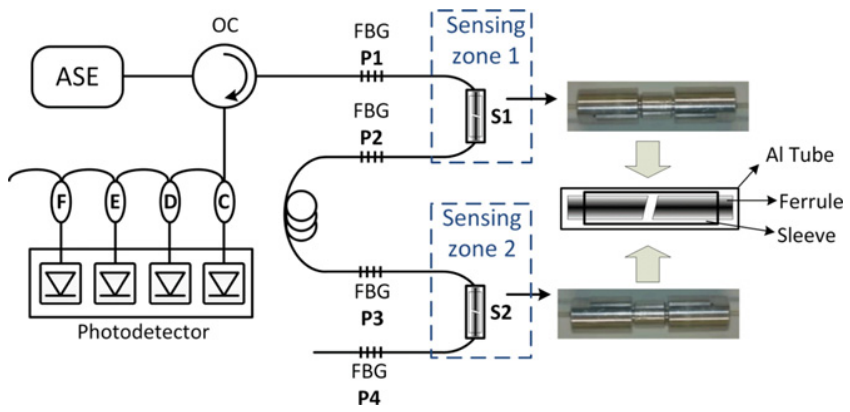

Fig. 1. Schematic of the experimental setup of the intensity-based temperature sensor. shown in the inset-photo of Fig. 1. The intensity-based sensing heads were located between two FBGs in order to provide a reference and a measurand signal. The reference signal was obtained by the ratio between the reflected optical power coming from the sensing and reference FBGs.

For the temperature measurements, the intensity-based sensing heads $\left(S_{1}\right.$ and $\left.S_{2}\right)$ were placed in a tubular oven built in-house, which permitted the temperature value to be set with an error smaller than $0.1{ }^{\circ} \mathrm{C}$. It must be pointed out that the FBGs were kept at a constant temperature and mechanically unstrained, outside the oven.

\section{Experimental results}

Four different wavelength optical signals were acquired by the PIN photodetectors. The first one comes from the reflection of the FBG $\left(P_{1}\right)$ located before the intensity sensing head $S_{1}$. Due to the implemented experimental setup, the optical intensity of this signal remains constant as heating occurs. The remaining signals were obtained from the other FBGs $\left(P_{2}, P_{3}\right.$ and $P_{4}$, respectively). This behaviour can be observed in Fig. 2, where the optical power responses of each FBG are presented when sensing head $S_{1}$ is at room temperature and when is heated up to $90{ }^{\circ} \mathrm{C}$. In this first experiment $S_{2}$ is kept at a constant temperature. From this figure, it can be verified that the amplitude of the signal only decreases in $P_{2}, P_{3}$ and $P_{4}$, as expected. This occurs due to the thermal expansion of the sensor $S_{1}$, which will induce a loss in the signal between the two ferrules, producing a variation in the amplitude of the optical power at the followings FBGs.

The same performance is noticed in Fig. 3, where sensing head $S_{2}$ was heated. It was observed that the optical power $P_{4}$ decreased with increasing temperature, corresponding to the thermal expansion of the sensor that occurs in sensor $S_{2}$. For high temperature, all the peaks of optical power decrease. This behaviour is due to a decrease of the optical source intensity between measurements.

Fig. 4 exhibits the response of the optical power ratio $P_{2} / P_{1}$ as the sensor $S_{1}$ is subjected to temperature variations. With the increase of temperature it is observable that the ratio between $P_{2}$ and $P_{1}$ decays. The temperature response has a behaviour that corresponds to a third order polynomial fit. The correspondent fitting equation is given by:

$$
\frac{P_{2}}{P_{1}}=-1.1 \times 10^{-2} T+1.28 \times 10^{-4} T^{2}-4.98 \times 10^{-7} T^{3}
$$
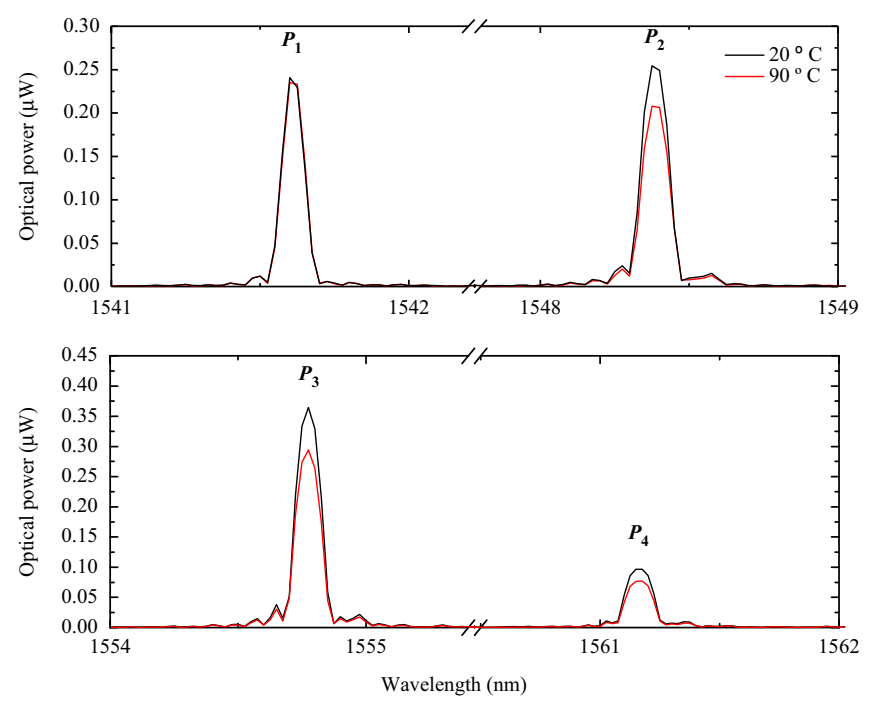

Fig. 2. Spectral response of the FBGs in reflection when $S_{1}$ experiences temperature variation and $S_{2}$ is kept at a constant temperature. 

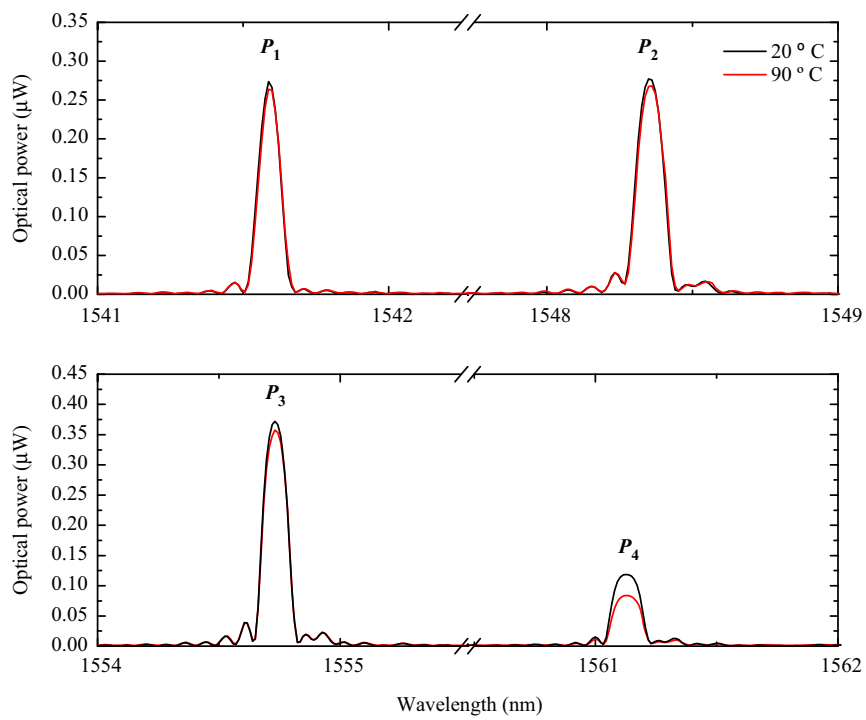

Fig. 3. Spectral response of the FBGs in reflection when $S_{1}$ is kept at a constant temperature and $S_{2}$ experiences temperature variation.

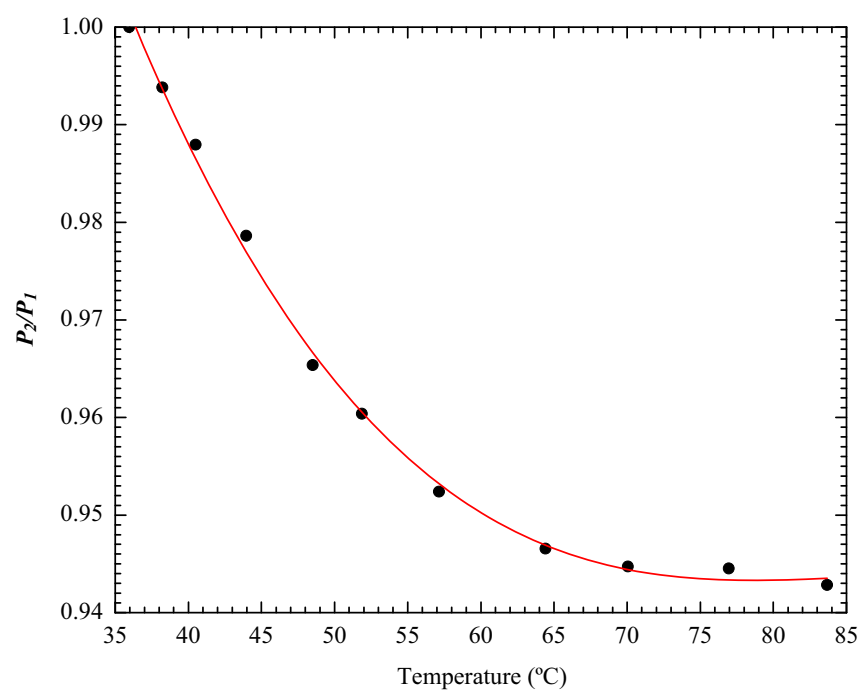

Fig. 4. Optical power ratio of peaks $P_{2}$ and $P_{1}$ response to temperature variation of the sensing head $S_{1}$.

Considering the temperature intervals of $[36,48.5]^{\circ} \mathrm{C}$ and $[64,85]{ }^{\circ} \mathrm{C}$, an approach to a linear-fitting behaviour can be done. In this case, the sensitivities obtained were $2.67 \times 10^{-3}{ }^{\circ} \mathrm{C}^{-1}$ and $1.74 \times 10^{-4}{ }^{\circ} \mathrm{C}^{-1}$, respectively. These variations are due to the thermal expansion of sensing head materials, namely the thermal expansion of the aluminium (external packaging), zirconia (ferrule connector), silica (optical fibre) and the epoxy (glue).

In Fig. 5 it is presented the relative error for the optical power ratio between the peaks $P_{2}$ and $P_{1}$ response to temperature variations of the FBG pair $\left(P_{1}, P_{2}\right)$. For these measurements $S_{1}$ was at room temperature. This result demonstrates that the response showed in Fig. 4 could be affected by a relative variation of about $\pm 0.7 \%$, which also reveals that the proposed selfreferenced scheme is effective on the temperature working range of the sensor head $S_{1}$.

Fig. 6 presents the amplitude ratio between the two peaks $P_{4}$ and $P_{3}$ when $S_{1}$ is subjected to temperature variation. The behaviour observed was as expected, since $S_{2}$ is kept at a constant temperature. The sensitivity achieved was $6.43 \times 10^{-6}{ }^{\circ} \mathrm{C}$. From the analysis of Fig. 6 , the crosstalk between the two sensing heads

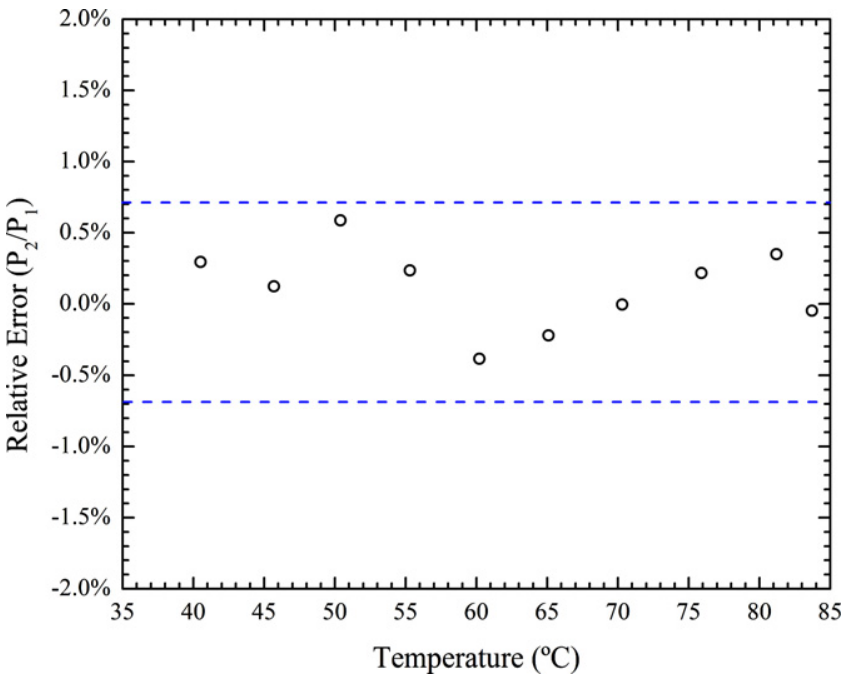

Fig. 5. Relative error in optical power ration of the peaks $P_{1}$ and $P_{2}$, when the FBG pair $\left(P_{1}, P_{2}\right)$ were subjected to temperature variations. $\left(S_{1}\right.$ was kept at constant temperature).

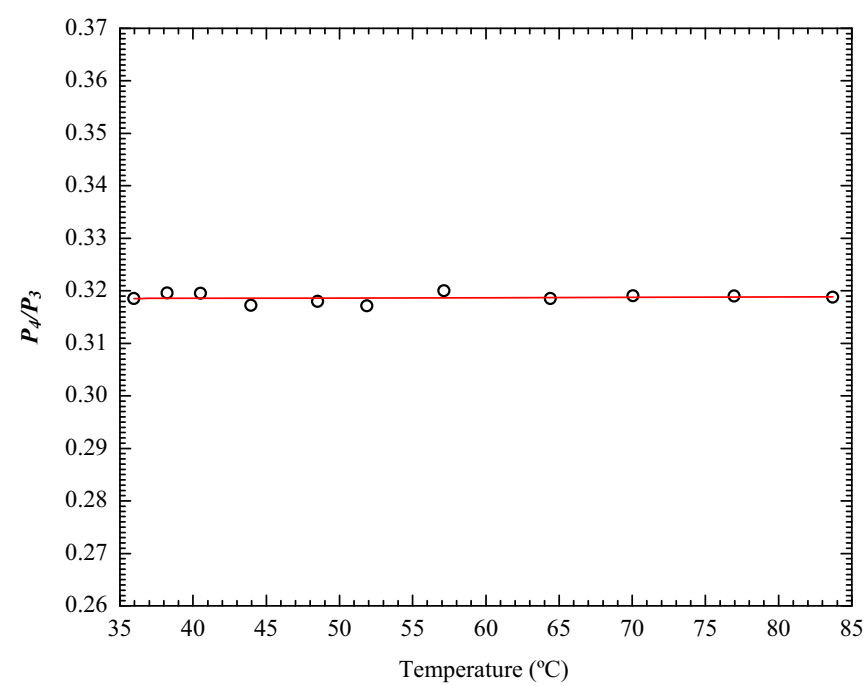

Fig. 6. Optical power ratio of peaks $P_{4}$ and $P_{3}$ response to temperature variation of the sensing head $S_{1}$.

although existent is negligible. The crosstalk is minimum when the FBG wavelength is located at the centre wavelength of the add-drop filter.

An estimation of how many sensors could be multiplexed with this sensing system was performed. The losses obtained due to the increase of the number of sensing heads were determined through the minimum losses of a single sensing head. Fig. 7 shows the relationship between number of the sensing heads and the losses variation obtained by the fabrication process. The arrow in the figure identifies the maximum of sensors that can be used in series, in this case it is possible to have twelve temperature optical fibre sensors. It should be noticed that is possible to implement more sensors, using for example optical amplification.

\section{Conclusions}

In summary, a simple network of two referenced intensitybased optical fibre sensors for temperature measurement was described. It was observed that the amplitude of the signal FBGs 


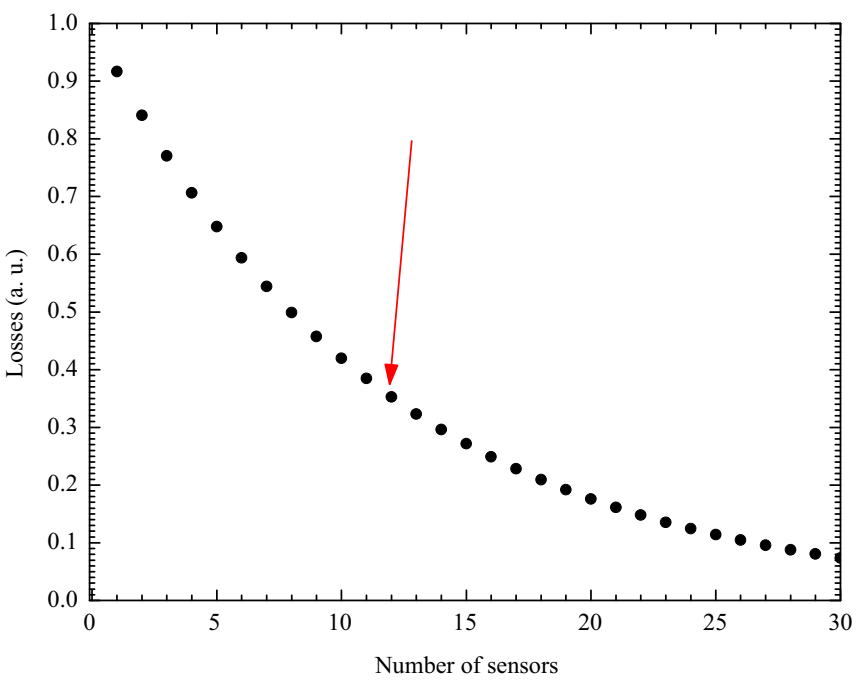

Fig. 7. Number of sensors used in series on one single fibre link.

decayed as temperature increased due to the thermal expansion that occurred in the sensing head. The sensor temperature response shows a behaviour that corresponds to a third order polynomial-fitting curve. The linear-sensitivities obtained to lower and higher temperature regions were $2.67 \times 10^{-3}{ }^{\circ} \mathrm{C}^{-1}$ and $1.74 \times 10^{-4}{ }^{\circ} \mathrm{C}^{-1}$, respectively. Relatively to the sensor network multiplexing capability, this type of sensing head can be arranged in a series network topology with an acceptable number of sensors, in this case, a network of twelve sensors located along a single fibre link without optical amplification, was estimated.

\section{References}

[1] E. Udd, Fiber Optic Sensors, Wiley Online Library, 1991.

[2] A. Wang, M.S. Miller, A.J. Plante, et al., Applied Optics 35 (15) (1996) 2595.

[3] T.C. Rozzell, C.C. Johnson, C.H. Durney, et al., Journal Microwave Power 9 (3) (1974) 241

[4] F. Mitschke, Optics Letters 14 (17) (1989) 967

[5] K. Bohnert, P. Gabus, J. Nehring, et al., Journal of Lightwave Technology 20 (2) (2002) 267.

[6] T. Tanaka, G.B. Benedek, Applied Optics 14 (1) (1975) 189.

[7] S. Abad, M. Lopez-Amo, F.M. Araujo, et al., Optics Letters 27 (4) (2002) 222.

[8] S.M. Melle, K.X. Liu, R.M. Measures, IEEE Photonics Technology Letters 4 (5) (1992) 516.

[9] S.M. Melle, K. Liu, Applied Optics 32 (19) (1993) 3601.

[10] M.A. Davis, A.D. Kersey, Electron Letters 30 (1) (1994) 75.

[11] P.M. Cavaleiro, A.B.L. Ribeiro, J.L. Santos, Electron Letters 31 (5) (1995) 392.

[12] A. Wilson, S.W. James, R.P. Tatam, Measurement Science and Technology 12 (2) (2001) 181. 